\title{
MMP-13 Enzyme and pH Responsive Theranostic Nanoplatform for Osteoarthritis
}

qiumei lan ( $\nabla$ qiumeilan33@163.com )

Guangxi Medical University

\section{Haimen Chen}

Guangxi Medical University

\section{Yunfen Pang}

Guangxi Medical University

\section{Feng Xiong}

Guangxi Medical University

\section{Chong Shen}

Guangxi Medical University

\section{Zainen Qin}

Guangxi Medical University

\section{Li Zheng}

Guangxi Medical University Jinmin Zhao

Guangxi Medical University

\section{Research}

Keywords: MMP-13/pH sensitive, cartilage targeting, osteoarthritis, theranostics

Posted Date: June 29th, 2020

DOI: https://doi.org/10.21203/rs.2.24580/v2

License: (c) (1) This work is licensed under a Creative Commons Attribution 4.0 International License. Read Full License

Version of Record: A version of this preprint was published on August 27th, 2020. See the published version at https://doi.org/10.1186/s12951-020-00666-7. 


\section{Abstract}

Stimulus-responsive therapy permits precise control of therapeutic effect only at lesion of interest, which determines it a promising method for diagnosis and imaging-guided precision therapy. The acid environment and overexpressed matrix metalloproteinases-13 (MMP-13) are typical markers in osteoarthritis (OA), which enables the development of stimulus-responsive drug delivery system with high specificity for OA. We herein demonstrate a nano-micelle based stimuli-responsive theranostic strategy with reporting and drug release controlled by acidic PH and MMP-13 for OA therapy. Such nanoplatform is incorporated with a motif specifically targeting on cartilage, a motif responsive to matrix metalloproteinases-13 to specifically report OA condition and biodynamics of nano-micelles, an antiinflammatory drug (e.g., psoralidin (PSO)) from traditional Chinese medicine, and a biocompatible polymeric skeleton for sustainable drug release in response to the acidic OA condition. The high effectiveness of this targeted precision therapy is demonstrated comprehensively by both in vitro and vivo evidences.

\section{Introduction}

As the most common chronic joint disease, osteoarthritis (OA) is regarded as the primary cause of disability among older adults[1,2]. Systemic administration and intra-articular injections of glucocorticoids or non-steroidal anti-inflammatory drugs (NSAID) are hard to exert maximize therapeutic benefit due to the avascular tissue on articular surface, the poor bioavailability and short half-life of drug[3], and rapid drug clearance[4]. Significant efforts have focused on engineering drug delivery systems to prolong the retention time of drug in the joint $[5,6]$. In recent years, emerging stimulusresponsive smart systems for targeted imaging and precision therapy have attracted increasing interest, such as $\mathrm{pH}[7,8], \mathrm{ROS}[9]$ or $\mathrm{NO}[10]$ triggered drug release systems upon exposure to inflammation tissues, which not only prolonged drug release, but also increased specificity to tissue and cells. The selection of optimal stimulus for the smart drug delivery system is the key for OA therapy.

During the process of $\mathrm{OA}$, the increase of active protease can degrade the cartilage ECM, which is considered as the main cause of cartilage destruction, such as matrix metalloproteinases (MMPs), adamalysin-like metalloproteinases with thrombospondin motifs (ADAMTSs), and cathepsins. Among them, matrix metallopeptidase 13 (MMP-13) that can cleave cartilage type Il collagen is considered as one of the major therapeutic targets. MMP-13 is highly expressed in the hyaline cartilage of OA patients in comparison to healthy cartilage[11]. With the alteration in the physical environment and release of inflammatory mediators by articular cells (under disease conditions), an acidity in the OA environment is generated $[12,13]$. The unique expressed MMP-13 $[14,15]$ and acidic $\mathrm{pH}[16]$ in $\mathrm{OA}$ joints enable the development of smart drug delivery system.

Poly (2-ethyl-2-oxazoline)-poly ( $\varepsilon$-caprolactone) (PEOz-PCL or PPL) is a biocompatible and biodegradable polymer[17]. It has been utilized to construct $\mathrm{pH}$-responsive drug carriers because protonation of the tertiary amine on PEOz backbone promotes endosome escape and drug release[18, 19]. Here, a specific 
collagen type II targeting peptide (Coll-II a1 chain-binding peptide - CollB) with the sequence of WRYGRL[20, 21] was grafted onto PPL to form cartilage targeting PPL (C-PPL). In parallel, PPL was conjugated with a specific peptide substrate of MMP-13 enzyme $\left(\mathrm{H}_{2} \mathrm{~N}-\mathrm{GPLGVRGC}-\mathrm{SH}\right)$ [22, 23]labeled with a fluorescence dye (Cy5.5). Further, black hole quencher-3 (BHQ-3) that can quench Cy5.5 fluorescence was coupled via amide reaction to obtain MMP-13 responsive and $\mathrm{pH}$ sensitive polymer MRCy5.5-BHQ-3-PPL (MR-PPL). Lastly, cartilage-targeting and OA-specific theranostic nanoplatform (MRC-PPL) was obtained by self-assembly of C-PPL and MR-PPL, and was further employed as the carrier to load a traditional Chinese medicine, psoralidin (PSO), one of the active ingredients isolated from Psoralea corylifolia that has therapeutic effects on cardiovascular and inflammatory diseases[24, 25].

As schematically illustrated in Figure 1, with intra-articular injection (IA) and guidance by CollB peptide, the multifunctional MRC-PPL@PSO nano-micelles specifically target on articular cartilage in the joint. Subsequently, abundant MMP-13 enzymes in OA environment cleave their substrate on the micelles, causing release of Cy5.5. The relief of quenching by BHQ-3 enables strong fluorescence signal from Cy5.5 whereby reporting the OA condition. Meanwhile, the acidic condition of OA leads to gradual disassembly of the micelles and consequent release of PSO molecules.

Figure 1. Schematic illustration of the synthesis and working mechanism of MMP-13 and pH responsive theranostic MRC-PPL@PSO nano-micelles for osteoarthritis.

\section{Results}

\subsection{Characterizations of MRC-PPL}

PPL copolymers self-assembled to nano-micelles $(120.3 \pm 22.81 \mathrm{~nm})$, as revealed by transmission electron microscopy (TEM, Figure 2a). Similarly, the hydrodynamic diameter reported by dynamic light scattering (DLS) is $121.5 \pm 26.1 \mathrm{~nm}$ (Figure $2 \mathrm{~b}$ ). And loading micelles with PSO molecules using thin-film hydration method didn't obviously alter the micelle size (Figure 2a). Zeta-potential measurements (Figure $2 \mathrm{c}$ ) indicate that micelles consisting of only PPL are negatively charged (-9.25 mV), while MRC-PPL micelles are less negative $(-5.38 \mathrm{mV})$. PSO is highly negatively charged $(-8.75 \mathrm{mV})$ which hinders its cellular uptake since cell membrane is also negatively charged[21,26]. Desirably, the negative charge of MRC-PPL@PSO micelles is significantly lesser $(-5.75 \mathrm{mV})$. The successful construction of MRC-PPL was further confirmed by the blue shift induced by incorporation of BHQ-3 and Cy5.5 in MRC-PPL in UV-vis spectra (Figure 2d).

Because of the energy resonance transfer to BHQ-3, the fluorescence intensity from Cy5.5 in MRC-PPL is much weaker than the equal amount of free Cy5.5 molecules (Figure 2e). As demonstrated in Figure 2f, introduction of MMP-13 enzyme to MRC-PPL dispersion led to gradual increases of fluorescence (becoming 3 times brighter after $30 \mathrm{~min}$ ), whereas the presence of MMP-13 inhibitor prevented this phenomenon. This observation confirms the MMP-13 specific (or OA-specific) fluorescence response, 
resulting from the cleavage of GPLGVRGC peptide in the probe and subsequent escape of Cy5.5 from quenching by BHQ-3.

\subsection{Drug release studies}

The strong hydrophobicity of PSO has significantly prevented its application in OA. But through hydrophobic interaction it can be readily loaded into the core of MRC-PPL micelle via self-assembly. The loading percentage of PSO in the micelles is as high as $16.9 \%$. The release of PSO from MRC-PPL@PSO at $\mathrm{pH}=7.4$ and $\mathrm{pH}=6.5$ was determined by high performance liquid chromatography (HPLC) in vitro. As shown in Figure $\mathbf{2 g}$, release of PSO was significantly accelerated by low pH $(6.9 \%$ at pH 7.4 vs. $21.1 \%$ at pH 6.5 within 48 h). Evidently, MRC-PPL@PSO enables effective and sustained drug release under the acidic OA condition.

Figure 2. Characterization of MRC-PPL nano-micelles. (a) TEM images of MRC-PPL and MRC-PPL@PSO micelles. Scale bare $=500 \mathrm{~nm}$. (b) Size distribution of MRC-PPL micelles based on dynamic light scattering. (c) Zeta potentials of PSO, PPL, MRC-PPL micelles and MRC-PPL@PSO micelles. (d) UV-Vis absorbance. (e) Fluorescence intensity of MRC-PPL micelles and Cy5.5. (f) Fluorescence intensity of MRC-PPL micelles, without or with MMP-13 $(0.01 \mu \mathrm{M})$, in the absence or presence of MMP-13 inhibitor $(0.45 \mu \mathrm{M})$. (g) In vitro release of PSO from MRC-PPL micelles in PBS ( $\mathrm{pH} 6.5$ and 7.4$)$ with $0.1 \%$ Tween 80 $($ mean $\pm S D, n=3)$.

\subsection{In vitro cytotoxicity assessment}

The cytotoxicity of micelles to chondrocytes isolated from C57BL6/J mice was investigated in vitro using CCK8 assay. Even at a high concentration $(200 \mu \mathrm{g} / \mathrm{mL})$ of MRC-PPL, the cell viability is $>85 \%$ after 48 hours of incubation, indicating its good biocompatibility (Figure 3a). Then, the possible cytotoxicity of PSO-loaded MRC-PPL micelles was determined as the function of PSO concentration. Figure $3 \mathbf{b}$ shows that MRC-PPL@PSO micelles carrying $15 \mu \mathrm{M}$ PSO didn't exert any cytotoxicity. Therefore, this dosage was chosen for the following experiments.

High level of interleukin 1 beta (IL-1 $\beta$ ) is a marker for $\mathrm{OA}$ and is commonly used to induce inflammation of chondrocytes[27-29]. As showed in Figure 3 c, IL-1 $\beta$ stimulation $(10 \mathrm{ng} / \mathrm{mL})$ for $48 \mathrm{~h}$ caused $40 \%$ decrease of cell viability while MRC-PPL@PSO treatment largely rescued IL-1 $\beta$ induced cell death. We then used quantitative real-time PCR (qRT-PCR) to detect the gene expression levels of TNF-a, MMP-3 and $M M P-13$ with promoting cartilage destruction effect in each treatment group, as well as the chondroidspecific marker, Col2a1. As shown in Figure 3d, the expressions of TNF-a, MMP-3 and MMP-13 are elevated by $115.72,3.33$ and 2.59 times respectively after IL-1 $\beta$ stimulation whereas the expression of Col2a1 was decreased to $23.78 \%$. The upregulation of inflammatory factors and down-regulation of Col2a1 were significantly reversed by MRC-PPL@PSO treatment.

As shown in hematoxylin and eosin (HE) staining (Figure 3e), chondrocytes with spindle shape and circular nucleate should be significantly transformed into elongated fibroblast-like cells after treatment of 
IL-1 $\beta$ for $24 \mathrm{~h}$. MRC-PPL@PSO was able to restore the morphology of most IL-1 $\beta$ pre-treated chondrocytes. MMP-13 and TNF-a that play important roles in OA development were detected by immunofluorescence staining. As shown in Figure 3e, positive staining in immunofluorescence staining (MMP-13 or TNF- $\alpha$ ) in IL-1 $\beta$-stimulated cells showed intense green fluorescence in IL-1 $\beta$ group. However, the fluorescence intensity was obviously decreased after treated by MRC-PPL@PSO.

Taking the observations in Figure 3ce together, we provide the in vitro evidence that MRC-PPL@PSO can efficiently rectify inflammation and rescue cartilage degradation in OA. PSO and MR-PPL @ PSO also play an anti-inflammatory role, but the effectiveness of PSO is much lower. MR-PPL@PSO micelles performed better than free PSO molecules because the engineered micelles can more effectively carry the hydrophobic drug into cells. MRC-PPL@PSO micelles outperformed MR-PPL@PSO micelles because it is equipped with cartilage targeting peptide.

Figure 3. In vitro study on chondrocytes isolated from C57BL6/J mice. (a and b) Cell viability after treatment with MRC-PPL or MRC-PPL@PSO. (c) Cell viability after various treatments to IL-1 $\beta$-stimulated chondrocytes. (d) Relative mRNA levels of Col2a1, TNF-a, MMP-3 and MMP-13 on IL-1 $\beta$-stimulated chondrocytes with various treatments. (e) HE staining and immunofluorescence images. The nuclei were counterstained with DAPI (blue), and MMP-13 or TNF-a positive staining was stained with FITC (green). Scales bar: $400 \mu \mathrm{m}$. Each data point represents mean $\pm \mathrm{s} . \mathrm{d}(\mathrm{n}=3)$. *, \# indicate $\mathrm{p}<0.05$; ${ }^{*}$, \#\# indicate $\mathrm{p}<$ $0.01 ; * \star \star$, \#\#\# indicate $p<0.001$.

2.4 Anti-inflammatory Effect of MRC-PPL@PSO on IL-1 $\beta$-Induced Chondrocytes via the MAPK, NF-KB, and $\mathrm{PI3K} /$ Akt Signaling Pathways.

During the progression of osteoarthritis, IL-1 $\beta$ levels are elevated, which activates the nuclear factor NFKB pathways to induce the expression of matrix metalloproteinases (MMPs) in cultured chondrocytes, leading to ECM degradation, abnormal bone metabolism and inflammatory disease[30, 31]. In addition, activated P38 MAPK has been reported to promote nuclear translocation of NF-kB[32]. Previous studies have found that PSO suppresses the expression of pro-inflammatory cytokines and chemokines as well as the expression of MMPs, which are the key regulators of cartilage destruction[33]. The PI3K/AKT signaling pathway also plays a crucial role in OA[34]. To explore the potential molecular mechanisms underlying the nano-platform treatment, the involvement of the MAPK, NF-KB and PI3K/Akt signaling pathways was investigated by Western blots. In vitro study showed that IL-1 $\beta$-induced increase of phospho-P38/P38 (p-P38/P38), phospho-Akt/Akt (p-Akt/Akt) and NF-kB expression was diminished by each treatment group. (Fig. 4a). Among all the treatment groups, MRC-PPL@PSO exhibited the greatest reduction of related proteins expression, superior to the PSO and MR-PPL@PSO groups. These results indicate that released PSO inhibits the production of inflammatory mediators in OA possibly mediated by the regulation of the PI3K/AKT pathway or MAPK cascades, leading to NF-KB inactivation.

Figure 4. Molecular mechanism of MRC-PPL@PSO. The expression of PI3K/AKT, MAPK and NF-KB signaling pathway proteins p-P38, P38, p-Akt, Akt and NF-KB was determined by (a) western blot and (c- 
d) quantification analysis. Each data point represents mean $\pm \mathrm{s} . d(n=3) .{ }^{*}$, \# indicate $p<0.05 ; * \star$, \#\# indicate $p<0.01 ; * \star \star$, \#\#\# indicate $p<0.001$.

\subsection{Cellular uptake of MRC-PPL}

Cellular uptake and MMP-13 responsive behaviors of MRC-PPL micelles were investigated after $24 \mathrm{~h}$ incubation with normal chondrocytes and IL-1 $\beta$ stimulated chondrocytes. As shown in Figure 5a, after administration of MRC-PPL, IL-1 $\beta$ stimulated cells exhibit much stronger fluorescence intensity (red) than normal chondrocytes cells, indicating that MRC-PPL micelles are efficient in response to excessive MMP13 induced by IL-1 $\beta$ to release cy5.5 due to the grafting of MMP-13 peptide substrate in MRC-PPL. Moreover, most red fluorescence emitted from cy5.5 overlaps with the positive dye type II collagen (green fluorescence), suggesting MRC-PPL can successfully target to type II collagen produced by chondrocytes compared with MR-PPL (Figure 5a). The results demonstrated that MRC-PPL is highly responsive to MMP-13 and can effectively target to type II collagen.

Figure 5. In vitro cellular uptake of MRC-PPL or MR-PPL micelles. (a) Immunofluorescence staining in chondrocytes to co-localized with collagen type II in the presence of MMP-13 or its inhibitor. The nuclei were counterstained with DAPI (blue) and collagen type II was stained with FITC (green). Scales bar: 400 $\mu \mathrm{m}$. (b) Fluorescence quantification of Cy5.5 after uptake of MRC-PPL or MR-PPL micelles by cells. Scale bars: $40 \mu \mathrm{m}$. $\left(\mathrm{n}=3\right.$; mean $\pm \mathrm{s} . \mathrm{d} ;{ }^{*}$, \# indicate $\mathrm{p}<0.05$, ${ }^{\star *}$, \#\# indicate $\mathrm{p}<0.01,{ }^{* \star *}$, \#\#\# indicate $\mathrm{p}<0.001$. $)$

\subsection{Living imaging of animals}

Two weeks after IA injection of papain solution, OA developed in the knee joint of mice, showing mild swelling and deformity. As demonstrated by in vivo fluorescence imaging (Figure 6a) and quantitative fluorescence intensity profile over time (Figure 6b), MRC-PPL micelles had more accumulation and better retention in the OA joints than MR-PPL micelles, benefitting from the cartilage targeting peptide. In contrast, MRC-PPL micelles poorly accumulated in normal joints or OA joints or OA joints treated with MMP-13 inhibitor, due to insufficient presence of MMP-13 enzymes to activate the theranostic nanoplatforms. At day 21, fluorescence was still obvious in the MRC-PPL treated OA joint, but not in other organs including lung, spleen, heart, liver, kidney (Figure 6c). The results of in vivo imaging are consistent with those of in vitro imaging. Taken together, we provide both in vitro and in vivo evidence that MRC-PPL micelles can specifically target on cartilage and label OA joint.

Figure 6. In vivo fluorescence imaging in normal or OA knees of mice. (a) Fluorescence imaging of OA joints injected with MRC-PPL, MRC-PPL+MMP-13 inhibitor, or MR-PPL at different time post-IA injection, as well as normal joint injected with MRC-PPL (excitation $=630 \mathrm{~nm}$, emission $=700 \mathrm{~nm}$ ). (b) The corresponding fluorescence intensity from $\mathrm{OA}$ joints at different times. (c) Ex vivo fluorescence imaging of heart, liver, spleen, lung, kidney, left knee and right knee at day 21 post-injection. $(n=5$, mean \pm s.d.).

\subsection{MRC-PPL@PSO nano-micelles attenuate the progression of OA}


After the mice receiving different treatments and being sacrificed at week 2 or 6 , the femoral condyle and tibial plateau were collected and evaluated according to the criteria described by Lydia Wachsmuth et al for the depth of erosion in articular cartilage[35]. Compared with the control (healthy) group, OA features represented by cartilage erosion and osteophyte formation and deterioration were found in PBS group (Figure7a). Osteophyte and surface lesion were significantly reduced at week 2 and 6 after administrating MRC-PPL@PSO, with OARSI score reduction of $78.59 \%$ and $89.42 \%$ respectively (Figure 7 b). Although with much lesser degrees, PSO and MR-PPL@PSO were also able to reduce the scores by $47.38 \%$ and $63.19 \%$ respectively at week 6 .

Figure 7. The macroscopic observation (a) and macroscopic score (b) of cartilage after IA-injection with PBS, PSO, MR-PPL@PSO and MRC-PPL@PSO for 2 and 6 weeks. Each data points represents mean \pm s.d. $(n=5) . *$, \# indicate $p<0.05 ; * *$, \#\# indicate $p<0.01 ; * \star *$, \#\#\# indicate $p<0.001$.

The cartilage tissues were then evaluated by hematoxylin-eosin (H\&E) staining and Safranin O-fast green staining. As shown in Figure 8a, the cartilage layer with surface roughness, vertical cracks, erosion, denudation and deformation were observed in the PBS group, which were consistent with the characteristics of OA. Compared with the PBS group, all three treated groups showed different degrees of improvement in morphological change, matrix staining and tidemark integrity. Noteworthy, MRCPPL@PSO can reduce the erosion and deformation of the cartilage layer surface, as well as the proliferation of tissue cells. Therefore, it is an effective method to maintain the columnar structure of cartilage. Furthermore, MRC-PPL@PSO group showed more intensive Safranin 0 staining (red) than other groups, indicating more secretion glycosaminoglycan (Figure 8a). This phenomenon indicates that MRCPPL@PSO facilitates deposition of glycosaminoglycan and attenuates cartilage matrix depletion and cartilage thinning. As presented in Figure $\mathbf{8 b}$, the OARSI scores based on histological analysis in all the treatment groups decreased to some extent compared with the PBS group, and the MRC-PPL@PSO group showed the lowest score with about $42.3 \%$ and $64.7 \%$ reduction at week 2 and 6 , respectively. The protein expression level of MMP-13 was also assessed by immunohistochemistry staining in the cartilage at week 2 or 6 . The MMP-13 positive staining in chondrocytes was observed to be dark brown in PBS group (Figure 8c). On the contrary, the expression level of MMP-13 decreased after different treatments, following the order of PSO > MR-PPL@PSO > MRC-PPL@PSO. Noteworthy, the expression of MMP-13 in the MRC-PPL@PSO treated joints was nearly identical to the healthy control.

Figure 8. Histological analyses of different treatments for 2 or 6 weeks. (a) H\&E (upper) and safraninO/fast green staining (lower) of cartilage sections after the treatments with PBS, PSO, MR-PPL@PSO and MRC-PPL@PSO. Scale bar $=1 \mathrm{~mm}$. (b) OARSI scores of articular cartilage after the treatments. Each data points represents mean \pm s.d. $(n=5) . *$, \# indicate $p<0.05 ; * \star$, \#\# indicate $p<0.01$; $* \star *$, \#\#\# indicate $p<$ 0.001. (c) Immunohistochemical staining of MMP-13 on cartilage sections after the treatments. Scale bar $=300 \mu \mathrm{m}$. 


\section{Discussion}

OA affected joints are presented by overexpressed MMP-13 and acidic microenvironment, which enables the development of 'smart' dual-stimuli-responsive probes for highly-efficient and controlled delivery and release of theranostic agents. Herein we demonstrated a theranostic nanoplatform for osteoarthritis, activated by microenvironment of acidic and MMP-13 enzyme overexpression in OA joints. The incorporated collagen type II binding peptide facilitates the targeting and retention in the joint. Such disease specific stimulus-responsive strategy improves the efficiency and minimizes the side-effects. Upon activation, the theranostic nano-micelles (MRC-PPL@PSO) produce fluorescence signal and sustainably release the anti-inflammatory drug molecules. In IL-1 $\beta$-treated chondrocytes, MRC-PPL@PSO promoted cell proliferation and inhibited inflammatory responses by down-regulating TNF- $a, M M P-3$ and $M M P-13$, and significantly alleviated the cartilage lesions with the lowest OARSI score, as confirmed by histological staining and MMP-13 expression after 2 and 6 weeks post-treatment, indicating the protective and the targeting therapeutic effect on cartilage. Furthermore, MRC-PPL@PSO exerts antiarthritic effects by regulation of PI3K/AKT, MAPK and NF-KB signaling pathway.

In principle, multiple drug compounds can be loaded to achieve synergistic effects. In addition, such biocompatible PEOz-PCL based polymeric system can be readily modified for diagnosis and treatment of other diseases (e.g., cancers) by varying the targeting motif, enzyme substrates, and drugs.

\section{Materials And Methods}

\subsection{Materials}

Poly( $\varepsilon$-caprolactone)-Poly(2-ethyl-2-oxazoline)-amine (PCL-PEOz- $\mathrm{NH}_{2}, \mathrm{Mw} 5000 \mathrm{Da}$ ) was purchased from Ruixibiotech (China). Psoralidin (PSO) and CCK8 were obtained from Sigma-Aldrich (US). Cy5.5-NHS and BHQ-3000S-5 was purchased from Seebio Biotech (China). N-Hydroxysuccinimide (NHS), N-(3dimethylaminopropyl)-N'-ethylcarbodiimide hydrochloride $(\mathrm{EDC} \cdot \mathrm{HCl})$ and $\mathrm{N}$-succinimidyl-4-maleimide butyrate (GMBS) were supplied by J\&K Scientific (China).

\subsection{Synthesis and characterizations of MRC-PPL micelles}

MMP-13/pH responsive and cartilage targeting MRC-PPL micelles was obtained by self-assembly of CPPL and MR-PPL[36]. Specifically, a Cy5.5 labeled MMP-13 substrate with the sequence of $\mathrm{H}_{2} \mathrm{~N}$ GPLGVRGC-SH was dissolved in DMSO $(15 \mathrm{mmol}, 500 \mu \mathrm{L})$ followed by the addition of N-succinimidyl-4Maleimidobutyrate (30 mmol in $100 \mu \mathrm{L} \mathrm{DMSO}$ ) to form MR-Cy 5.5. The reaction solution was incubated at room temperature for $4 \mathrm{~h}$ in a $1.5 \mathrm{~mL}$ eppendorf tube with gentle shaking. Then, to conjugate with the MMP-13 responsive peptide, EDC and NHS were added in MR-Cy5.5 solution, followed by the addition of 50 mg PPL. Subsequently, BHQ-3 was added in dark to form MRCy5.5-BHQ-3-PPL (MR-PPL), followed by the purification using HPLC. In parallel, WYRGRL peptide (10 mg in $100 \mu \mathrm{L}$ DMSO) which specifically binds with collagen type II was coupled with PPL (50 mg in $100 \mu \mathrm{L} \mathrm{DMSO}$ ) to obtain C-PPL. With addition of $\mathrm{N}, \mathrm{N}$-diisopropyl ethylamine (DIPEA, $10 \mu \mathrm{L}$ ) and overnight reaction, MRC-PPL micelles self-assembled 
from C-PPL and MR-PPL were finally obtained after ultrasonication and filtration through a $0.8-\mu \mathrm{m}$ membrane.

Polymeric micelles can encapsulate drug compounds using the film hydration method[37, 38]. The PPL copolymer $(10 \mathrm{mg})$ and PSO $(1 \mathrm{mg})$ were dissolved in acetonitrile and then evaporated in a vacuum at

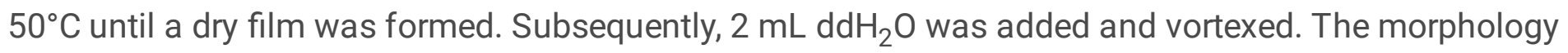
of MRC-PPL was uncovered by a transmission electron microscope (TEM, H-7650). We used Malvern Zetasizer Nano ZS90 to measure the size distribution and zeta potential of the micelles in aqueous suspension. Absorption spectra were obtained from a microplate reader (Thermo Scientific Multiskan GO Microplate Spectrophotometer). And the fluorescence from Cy5.5 was detected using a Fluorescence spectrophotometer (RF-5301PC).

The loading capability of PSO in MRC-PPL micelles was determined using the HPLC. Briefly, Lyophilized MRC-PPL@PSO powder was dissolved in $\mathrm{HCl}(0.1 \mathrm{~mol} / \mathrm{L})$ and moderate methanol was added to release PSO. After the supernatant was collected by centrifugation (6000 rpm,30 minutes), the PSO concentration in the supernatant was detected by ACQ-BSM HPLC system (Waters, Milford, MA, USA).The system parameters were set to wavelength $347 \mathrm{~nm}$, and the $\mathrm{C} 18$ analytical column $(250 \times 4.6 \mathrm{~mm}, 5$ microns) was used at $37^{\circ} \mathrm{C}$. The mobile phase was consisted of methanol/water/0.05 $\mathrm{M}$ acetic acid $(78 / 22 / 0.2, \mathrm{v} / \mathrm{v})$ and the flow rate was $1.0 \mathrm{~mL} / \mathrm{min}$. The loading content (LC) of PSO were calculated according to the formula:

LC $(\%)=($ the mass of loaded PSO / the total mass of nanoparticles $) \times 100 \%$

\subsection{Drug release studies}

The pH-dependent release behavior of PSO from the MRC-PPL@PSO micelles was monitored at $37^{\circ} \mathrm{C}$. Briefly, $2 \mathrm{~mL}$ of the PSO-loaded micelles were placed in a dialysis bag (MWCO 3500), which was then immersed in PBS (10 mM, pH 6.5 and 7.4) containing $1 \%$ Tween 80 . The release device was continuously shaken at $37^{\circ} \mathrm{C}$. Remove $1 \mathrm{~mL}$ of release media at predetermined intervals and immediately replace it with $1 \mathrm{~mL}$ of fresh media. The accumulative release amount of PSO was finally determined by HPLC.

\subsection{The chondrocytes extraction and culture.}

The chondrocytes was extracted from the knee joints of new born (3-5 days) C57BL6/J mice (Animal Resources Center of Guangxi Medical University, Nanning, Guangxi) after the articular cartilage were minced, digested with trypsin for 30 minutes, and digested with type II collagenase ( $2 \mathrm{mg} / \mathrm{mL}, \mathrm{Gibco})$. Chondrocytes were cultured in Eagle medium (Gibco) containing1\% ( $/ / \mathrm{v}$ ) penicillin/streptomycin (Solarbio) and 10\% (v/v) fetal bovine serum (Gibco). Cell experiments were constructed according to our previous work.

\subsection{Cytotoxicity test}


The cytotoxicity of different formulations to chondrocytes cells were using Cell Counting Kit-8 (CCK-8; Dojindo Laboratories, Kumamoto, Japan). Briefly, the cell at a density of 5,000 per well were seeded in 96well plate overnight. Thereafter, MRC-PPL@PSO with the various amount of PSO was added and further incubated for $48 \mathrm{~h}$. MRC-PPL micelles without PSO at different concentrations ( $0 \sim 200 \mu \mathrm{L} / \mathrm{mL})$ were also tested for comparison. After treatment, $10 \mu \mathrm{L}$ of CCK-8 were added to each well and cultivated for $4 \mathrm{~h}$. Finally, the absorbance at $450 \mathrm{~nm}$ was measured using a microplate reader (Thermo Scientific Multiskan GO Microplate Spectrophotometer). The viability of MRC-PPL@PSO on the proliferation of IL-1 $\beta$-induced chondrocytes was measured by CCK8 assay. Chondrocytes with density of $1.5 \times 10^{4}$ cells/wells were cultured in 24-well plates, which were treated with $10 \mathrm{ng} / \mathrm{mL} \mathrm{IL-1} \beta$ for $2 \mathrm{~h}$ to establish an in vitro model of osteoarthritis. Incubated with the PSO (15 $\mu \mathrm{M})$, MR-PPL@PSO or MRC-PPL@PSO for 24 h. The latter two treatments equivalently contained $15 \mu \mathrm{M}$ PSO. The remaining steps are based on the CCK8 method.

\subsection{In vitro cellular uptake}

Cellular uptake of MRC-PPL was examined in chondrocytes. IL-1 $(10 \mathrm{ng} / \mathrm{mL})$ treated cells were added MRC-PPL or MR-PPL ( $1 \mathrm{mg} / \mathrm{mL})$. Meanwhile, the cells were stained with immunofluorescence against COL2A1 (Boster, Wuhan, China, 1:200). After the nuclei were stained with DAPI, the images were captured using a laser scanning confocal microscope (Nikon A1, Tokyo, Japan). The excitation wavelengths for DAPI and Cy5.5 were $405 \mathrm{~nm}$ and $640 \mathrm{~nm}$, respectively.

\subsection{In vitro anti-inflammatory activity and Immunofluorescence staining.}

The chondrocytes were seeded in 6-well (density of $5 \times 10^{4}$ cells $/ \mathrm{cm}^{2}$ ) or 24-well plates (density of $2 \times 10^{4}$ cells $\left./ \mathrm{cm}^{2}\right)$. The cells pretreated with IL-1 $\beta(10 \mathrm{ng} / \mathrm{mL})$ for $2 \mathrm{~h}$ were incubated with the PSO $(15 \mu \mathrm{M})$, MRPPL@PSO or MRC-PPL@PSO for 24 h. The latter two treatments equivalently contained $15 \mu \mathrm{M}$ PSO. The cells were then washed with saline and fixed with $95 \%$ ethyl alcohol for 30 minutes, followed by staining with hematoxylin and eosin (HE, Nanjing Jian Biotechnology, China).The immunofluorescence was used to detect the protein expression level of MMP-13 and TNF-a. Samples were incubated with primary antibodies against MMP-13 (Boster, China; 1:100) and TNF- $a$ (Boster, China; $1: 100$ ) at $37^{\circ} \mathrm{C}$ for $4 \mathrm{~h}$. The second antibody FITC-anti-rabbit IgG (Boster, China) was incubated in dark for $1 \mathrm{~h}$. Finally, the nuclei were stained with DAPI, and the cells were observed with fluorescence microscope (Olympus, Japan).

\section{8 qRT-PCR detection}

RNA separation kits (Megentec, China) were used to extract total RNA. The reverse transcription is done by a reverse transcription kit (Fermentas Company, USA). All qRT-PCR reactions were performed by a light Cycle 96 system (Roche, Switzerland) for $10 \mathrm{~min}$ at $95^{\circ} \mathrm{C}$, followed by 40 cycles with 10 s duration at 95 ${ }^{\circ} \mathrm{C}$ and then $60 \mathrm{~s}$ duration at $60^{\circ} \mathrm{C}$. The primers were used as follows in the table 1 :

Table 1. Primers for RT-PCR performance 


\begin{tabular}{llll} 
gene & Forward primer $\left(5^{\prime}-3^{\prime}\right)$ & Reverse primer $\left(5^{\prime}-3^{\prime}\right)$ & size \\
\hline TNF-a & CAGAAAGCATGATCCGCGAC & TTCACCCACATCAGGCACTC & 20 \\
\hline MMP-13 & TACCATCCTGCGACTCTTGC & TTCACCCACATCAGGCACTC & 20 \\
\hline MMP-3 & GTTCTGGGCTATACGAGGGC & TTCTTCACGGTTGCAGGGAG & 20 \\
\hline Col2a1 & ACACCGCTAACGTCCAGA TG & TCGGTACTCGATGACGGTCT & 20 \\
\hline B-actin & CCCATCTATGAGGGTTACGC & TTtAATGTCACGCACGATTTC & 20
\end{tabular}

\subsection{Western blot analysis}

Proteins were extracted from chondrocytes with RIPA lysis buffer. Lysates were sonicated on ice and centrifuged at $12000 \mathrm{rpm}$ for $30 \mathrm{~min}$ at $4{ }^{\circ} \mathrm{C}$. The protein concentration of the supernatant was determined using a BCA protein assay kit. Equal amounts of protein ( $40 \mu \mathrm{g})$ were separated by $10 \%$ SDSPAGE and transferred to PVDF membranes. The membranes were incubated with blocking buffer $5 \%$ nonfat milk in TBS containing $0.1 \%$ Tween-20 (TBST) for $2 \mathrm{~h}$ at room temperature and then probed with the primary antibodies against p65, Akt and p-Akt, P38, p-P38 (dilution 1:1000) overnight at $4{ }^{\circ} \mathrm{C}$. After washing three times with TBS containing $0.1 \%$ Tween-20 for $5 \mathrm{~min}$, the membranes were then incubated with the secondary antibody (Invitrogen, USA) and visualized using the Odyssey Infrared Imaging System (LI-COR USA) according to the manufacturer's instructions. Data were normalized by housekeeping protein (GAPDH).

\subsection{Induction of osteoarthritis in mice}

A total of $100 \mathrm{C57BL} / 6 \mathrm{~J}$ mice (aged 6-10 weeks) were used for the in vivo experiments, which were performed in accordance with the ethical approval of the Institutional Ethics Committee of Guangxi Medical University. Arthritis was induced by articular injection of a mixture of papain solution (Sigma; $8 \%$ W/V) with L-cysteine (Sigma, $0.03 \mathrm{~mol} / \mathrm{L}$ ) three times a week. Mice were randomly divided into different experimental groups $(n=5)$ : healthy control group, OA group and treatment groups. For OA group, mice were IA injected with $20 \mu \mathrm{L}$ of PBS once per week. The treatments included IA injection of $20 \mu \mathrm{L}$ PSO (15 $\mu \mathrm{M}$ ), $20 \mu \mathrm{L}$ MR-PPL@PSO solution (containing $15 \mu \mathrm{M}$ PSO), or $20 \mu \mathrm{L}$ MRC-PPL@PSO (containing $15 \mu \mathrm{M}$ PSO), once per week for 2 or 6 weeks.

\subsection{In vivo near infrared fluorescence (NIRF) imaging}

After anesthetized with $2 \%$ isoflurane (Aerrane; Baxter), mice were injected with treatment solution through the articular cavity ( $\mathrm{n}=5$ for each group). In vivo fluorescence imaging was performed on an IVIS 200 system with a Cy5.5 filter set (excitation $630 \mathrm{~nm}$; emission $695 \mathrm{~nm}$ ). The mice were anesthetized and imaged at 30min, 0.5, 1, 3, 7, 14, $21 \mathrm{~d}$ after injection.

\subsection{Ex-vivo NIRF imaging and histological analysis}


At research endpoints, mice were euthanized and their hind knee joints were collected for fluorescence imaging by an IVIS-200 system. The treated articular cartilages were harvested. Grading of articular cartilage surface was performed by specifically evaluating a nine-area grid of each medial and lateral tibia plateau (scale of 0-8)[35]. Moreover, the treated joints were fixed with $4 \%$ formaldehyde for 48 hours and decalcified with EDTA buffer. The samples were then embedded in paraffin and cut into sections of 3 microns. Paraffin sections were dewaxed and then collected for staining with hematoxylin (Solarbio, China). Glycosaminoglycan (GAG) secretion was detected using a safranin O-fast green kit (Solarbio, China) and MMP-13 secretion was assessed using a immunohistochemistry kit (Bioss, China). The stained slides were then imaged to examine the knee joint morphology on a fluorescence microscope. The OARSI cartilage OA histopathology grading system was adopted to evaluate the treated tissues[39].

\subsection{Statistical analysis}

All data were reported as mean \pm standard deviation of at least three experiments. The two-sided student $t$ test was used for statistical analysis. $P$ value less than or equal to 0.05 was considered statistically significant.

\section{Declarations}

\section{Ethics approval and consent to participate}

Animal care was in accordance with institutional guidelines. All animal studies were performed in accordance with the ethical approval of the Institutional Ethics Committee of Guangxi Medical University.

\section{Consent for publication}

Not applicable.

\section{Availability of data and materials}

The datasets used and/or analyzed during the current study are available from the corresponding author on reasonable request.

\section{Competing interests}

The authors declare no conflict of interest.

\section{Funding}

This study was financially supported by National Key R\&D Program of China(2018YFC1105900), National Natural Science Fund of China (Grant No. 81960414), the Guangxi Science and Technology Base and Talent Special Project (Grant No. GuikeAD17129012), the local Science and Technology Development Project leading by the central government (the three-D printing and digital medical platform, Grant No. GuikeZY18164004), the Basic ability enhancement project for young and middle-aged teachers 
of universities in Guangxi (2019KY0134 and 2018KY0127), and the Innovative Project of Postgraduate Majored in Biomedical Engineering (SG2018005 and SG2018007).

Authors' contributions

Q.M.L., Z.N.Q., L.Z. and J.M.Z. designed experiments and wrote the manuscript. Q.M.L., H.M.C., and Z.N.Q. synthesized and analyzed MRC-PPL micelles. Q.M.L. conducted the vitro experiments and histopathological analysis. Y.F.P., F.X. and C.S. contributed to animal studies and subsequent analysis. All authors read and approved the final manuscript.

Acknowledgements

Not applicable.

\section{References}

1. Dunlop DD, Manheim LM, Jing S, Chang RW: Arthritis prevalence and activity limitations in older adults. Arthritis \& Rheumatism 2001, 44:212-221.

2. Bender B, Perry M, Ramsey F, Boeselager G, Jackson-Thompson J: Prevalence and impact of chronic joint symptoms - Seven states, 1996 (Reprinted from MMWR, vol 47, pg 345-351, 1998). Journal of the American Medical Association 1998, 279:1940-1941.

3. Brown TJ, Laurent UB, Fraser: Turnover of hyaluronan in synovial joints: elimination of labelled hyaluronan from the knee joint of the rabbit. Experimental Physiology 1991, 76.

4. Albert C, Brocq O, Gerard D, Roux C, Euller-Ziegler L: Septic knee arthritis after intra-articular hyaluronate injection: Two case reports. Joint Bone Spine 2006, 73:205-207.

5. Bajpayee AG, Scheu M, Grodzinsky AJ, Porter RM: A rabbit model demonstrates the influence of cartilage thickness on intra-articular drug delivery and retention within cartilage. Journal of Orthopaedic Research 2015, 33:660-667.

6. Butoescu N, Jordan O, Burdet P, Stadelmann P, Petri-Fink A, Hofmann H, Doelker E: Dexamethasonecontaining biodegradable superparamagnetic microparticles for intra-articular administration: physicochemical and magnetic properties, in vitro and in vivo drug release. European Journal of Pharmaceutics \& Biopharmaceutics 2009, 72:529-538.

7. Li C, Li H, Wang Q, Zhou M, Li M, Gong T, Zhang Z, Sun X: pH-sensitive polymeric micelles for targeted delivery to inflamed joints. Journal of Controlled Release 2017, 246:133-141.

8. Dual-functional lipid polymeric hybrid pH-responsive nanoparticles decorated with cell penetrating peptide and folate for therapy against rheumatoid arthritis. European Journal of Pharmaceutics \& Biopharmaceutics Official Journal of Arbeitsgemeinschaft Fur Pharmazeutische Verfahrenstechnik E V2018.

9. Ming-Fan C, Wei-Tso C, Wei-Lin W, Yu-Jung L, Hsing-Wen S: Controlled Release of an Antiinflammatory Drug Using an Ultrasensitive ROS-Responsive Gas-Generating Carrier for Localized 
Inflammation Inhibition. Journal of the American Chemical Society 2015, 137:12462.

10. Jin P, Wiraja C, Zhao J, Zhang J, Zheng L, Xu C: Nitric oxide nanosensors for predicting the development of osteoarthritis in rat model. Acs Appl Mater Interfaces 2017, 9:25128-25137.

11. Meng F, Zhang Z, Chen W, Huang G, Liao W: MicroRNA-320 regulates matrix metalloproteinase-13 expression in chondrogenesis and interleukin-1 $\beta$-induced chondrocyte responses. Osteoarthritis \& Cartilage 2016, 24:932-941.

12. Goldie I, Nachemson A: Synovial pH in rheumatoid knee-joints. I. The effect of synovectomy. Acta Orthop Scand 1969, 40:634-641.

13. Chung MF, Chia WT, Wan WL, Lin YJ, Sung HW: Controlled Release of an Anti-inflammatory Drug Using an Ultrasensitive ROS-Responsive Gas-Generating Carrier for Localized Inflammation Inhibition. J Am Chem Soc 2015, 137:12462-12465.

14. Rousseau JC, Delmas PD: Biological markers in osteoarthritis. Nature Clinical Practice Rheumatology 2007, 3:346-356.

15. Neuhold LA, Killar L, Zhao W, Sung M-LA, Warner L, Kulik J, Turner J, Wu W, Billinghurst C, Meijers T: Postnatal expression in hyaline cartilage of constitutively active human collagenase-3 (MMP-13) induces osteoarthritis in mice. Jclininvest 2001, 107:35-44.

16. Chung M-F, Chia W-T, Wan W-L, Lin Y-J, Sung H-W: Controlled Release of an Anti-inflammatory Drug Using an Ultrasensitive ROS-Responsive Gas-Generating Carrier for Localized Inflammation Inhibition. Journal of the American Chemical Society 2015, 137:12462.

17. Qiu LY, Yan L, Zhang L, Jin YM, Zhao QH: Folate-modified poly(2-ethyl-2-oxazoline) as hydrophilic corona in polymeric micelles for enhanced intracellular doxorubicin delivery. Int J Pharm 2013, 456:315-324.

18. Wang D, Zhou Y, Li X, Qu X, Deng Y, Wang Z, He C, Zou Y, Jin Y, Liu Y: Mechanisms of pH-Sensitivity and Cellular Internalization of PEOz-b-PLA Micelles with Varied Hydrophilic/Hydrophobic Ratios and Intracellular Trafficking Routes and Fate of the Copolymer. Acs App/ Mater Interfaces 2017, 9:69166930.

19. Li C, Zhang X, Chen Q, Zhang J, Li W, Hu H, Zhao X, Qiao M, Chen D: Synthetic Polymeric Mixed Micelles Targeting Lymph Nodes Trigger Enhanced Cellular and Humoral Immune Responses. ACS Appl Mater Interfaces 2018, 10:2874-2889.

20. Rothenfluh DA, Bermudez H, O'Neil CP, Hubbell JA: Biofunctional polymer nanoparticles for intraarticular targeting and retention in cartilage. Nature Materials 2008, 7:248.

21. Formica FA, Barreto G, Zenobi-Wong M: Cartilage-targeting dexamethasone prodrugs increase the efficacy of dexamethasone. J Control Release 2019, 295:118-129.

22. Duro-Castano A, Lim NH, Tranchant I, Amoura M, Beau F, Wieland H, Kingler O, Herrmann M, Nazaré M, Plettenburg O, et al: In Vivo Imaging of MMP-13 Activity Using a Specific Polymer-FRET Peptide Conjugate Detects Early Osteoarthritis and Inhibitor Efficacy. Advanced Functional Materials 2018, 28:1802738. 
23. Lee S, Park K, Lee S-Y, Ryu JH, Park JW, Ahn HJ, Kwon IC, Youn I-C, Kim K, Choi K: Dark Quenched Matrix Metalloproteinase Fluorogenic Probe for Imaging Osteoarthritis Development $\backslash \mathrm{r}$ <i>in Vivo</i>. Bioconjugate Chemistry 2008, 19:1743-1747.

24. Rao Z, Wang S, Wang J: Protective effects of psoralidin on IL-1 $\beta$-induced chondrocyte apoptosis. Molecular Medicine Reports 2018, 17:3418.

25. Xin Z, Wu X, Yu Z, Shang J, Xu B, Jiang S, Yang Y: Mechanisms explaining the efficacy of psoralidin in cancer and osteoporosis, a review. Pharmacol Res 2019, 147:104334.

26. Hu Q, Chen Q, Yan X, Ding B, Chen D, Cheng L: Chondrocyte affinity peptide modified PAMAM conjugate as a nanoplatform for targeting and retention in cartilage. Nanomedicine 2018, 13:749767.

27. Xu X-X, Zhang X-H, Diao Y, Huang Y-X: Achyranthes bidentate saponins protect rat articular chondrocytes against interleukin-1 $\beta$-induced inflammation and apoptosis in?vitro. Kaohsiung Journal of Medical Sciences 2017, 33:62.

28. Zhang XH, XU X-X, XU T: Ginsenoside Ro suppresses interleukin-1 $\beta$-induced apoptosis and inflammation in rat chondrocytes by inhibiting NF-kB. Chinese Journal of Natural Medicines 2015, 13:283-289.

29. Feng Z, Zheng W, Li X, Lin J, Xie C, Li H, Cheng L, Wu A, Ni W: Cryptotanshinone protects against IL$1 \beta$-induced inflammation in human osteoarthritis chondrocytes and ameliorates the progression of osteoarthritis in mice. International Immunopharmacology 2017, 50:161-167.

30. Ying X, Chen X, Cheng S, Shen Y, Peng L, Xu HZ: Piperine inhibits IL- $\boldsymbol{\beta}$ induced expression of inflammatory mediators in human osteoarthritis chondrocyte. International Immunopharmacology 2013, 17:293-299.

31. Pulai JI, Chen H, Im HJ, Kumar S, Hanning C, Hegde PS, Loeser RF: NF-kB Mediates the Stimulation of Cytokine and Chemokine Expression by Human Articular Chondrocytes in Response to Fibronectin Fragments. Journal of Immunology 2005, 174:5781-5788.

32. Papachristou DJ, Papadakou E, Basdra EK, Baltopoulos P, Panagiotopoulos E, Papavassiliou AG: Involvement of the p38 MAPK-NF-KB Signal Transduction Pathway and COX-2 in the Pathobiology of Meniscus Degeneration in Humans. Molecular Medicine 2008, 14:160-166.

33. Rao Z, Wang S, Wang J: Protective effects of psoralidin on IL1betainduced chondrocyte apoptosis. Mol Med Rep 2018, 17:3418-3424.

34. Jia GH, Xia C, Xin PZ, Yi TT, Xiao YW, Gang S, Bing Z: 17ß-Estradiol promotes cell proliferation in rat osteoarthritis model chondrocytes via PI3K/Akt pathway. Cellular \& Molecular Biology Letters 2011, 16:564-575.

35. Wachsmuth L, Keiffer R, Juretschke H-P, Raiss RX, Kimmig N, Lindhorst E: In vivo contrast-enhanced micro MR-imaging of experimental osteoarthritis in the rabbit knee joint at 7.1T. Osteoarthritis Cartilage 2003, 11:891-902.

36. Lei Z, Jin X, Magdalena S, Fan Z, Qimeng Q, Ying M, Xuexun F, Kwangmeyung K, Seulki L, Xiaoyuan C: Real-Time Video Imaging of Protease Expression In Vivo. Theranostics 2011, 1:18-27. 
37. Wang Q, Jiang J, Chen W, HaoJiang, Zhang Z, Sun X: Targeted delivery of low-dose dexamethasone using PCL-PEG micelles for effective treatment of rheumatoid arthritis. Journal of Controlled Release 2016:S0168365916301821.

38. Zhan C, Gu B, Xie C, Li J, Liu Y, Lu W: Cyclic RGD conjugated poly(ethylene glycol)-co-poly(lactic acid) micelle enhances paclitaxel anti-glioblastoma effect. Journal of Controlled Release Official Journal of the Controlled Release Society 2010, 143:136-142.

39. Pritzker KPH, Gay S, Jimenez SA, Ostergaard K, Pelletier J-P, Revell PA, Salter D, Berg WBvd: Osteoarthritis cartilage histopathology: grading and staging. Osteoarthritis \& Cartilage 2006, 14:1329.

\section{Figures}
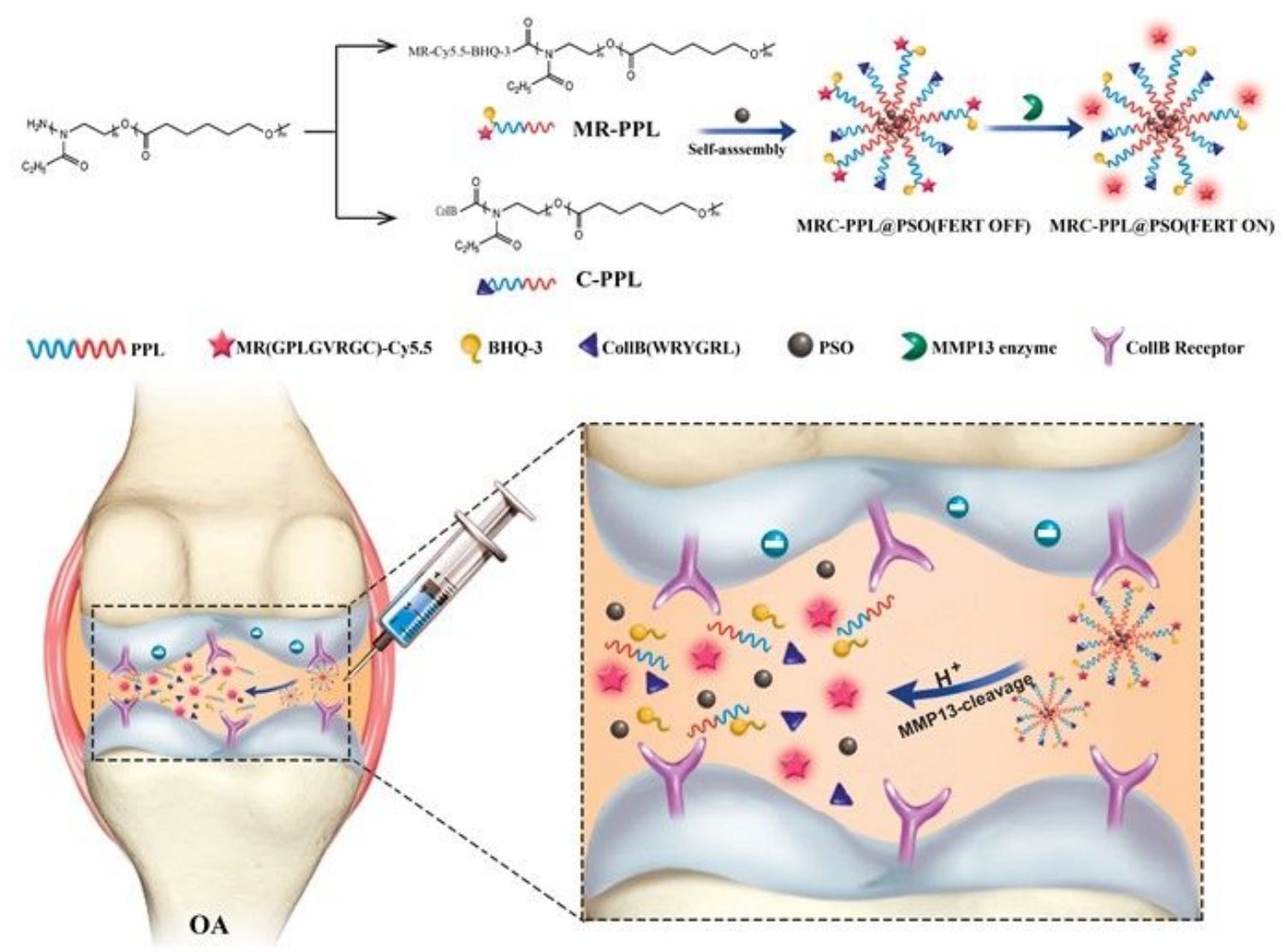

Figure 1

Schematic illustration of the synthesis and working mechanism of MMP-13 and pH responsive theranostic MRC-PPL@PSO nano-micelles for osteoarthritis. 
a
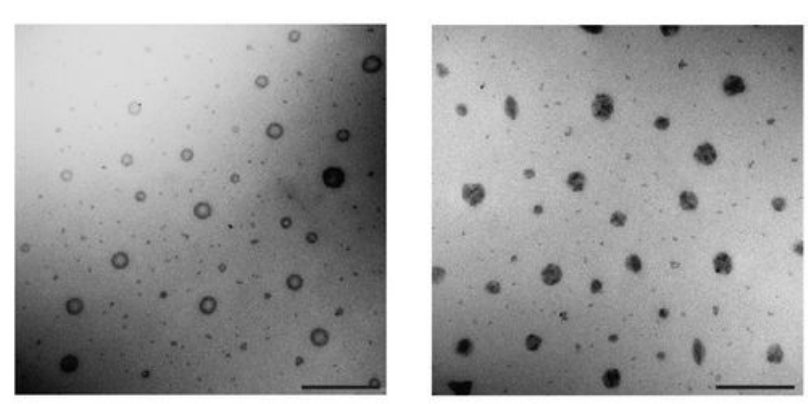

d

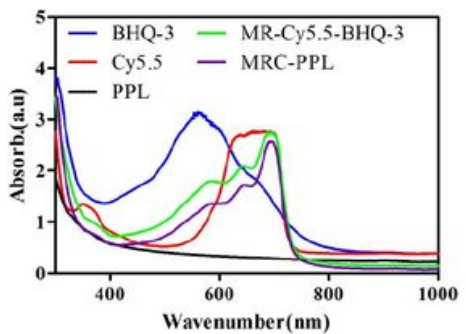

e

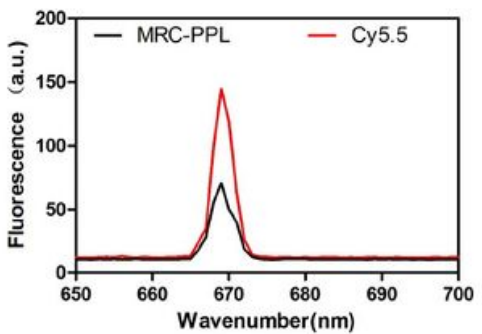

b

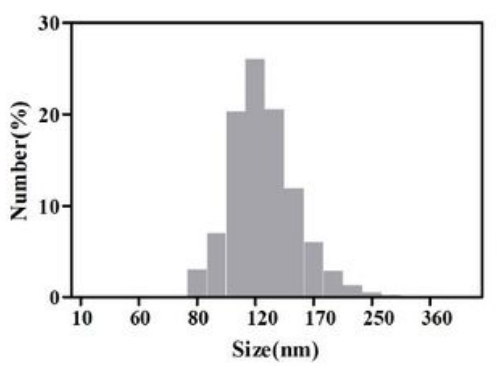

f

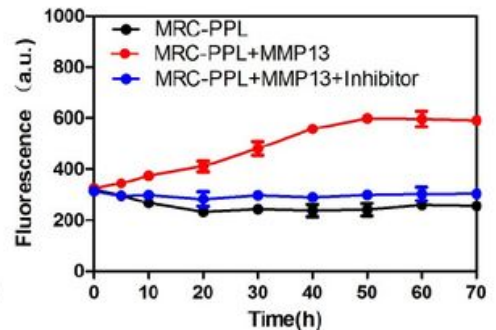

c

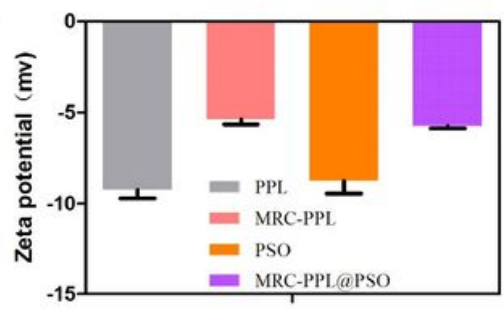

g

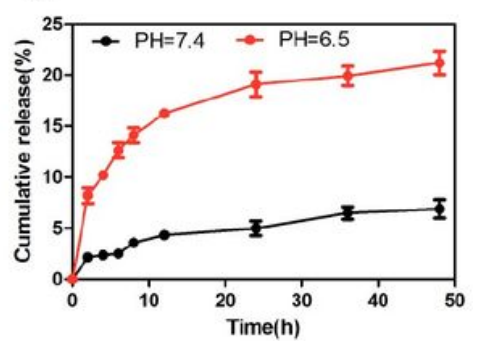

Figure 2

Characterization of MRC-PPL nano-micelles. (a) TEM images of MRC-PPL and MRC-PPL@PSO micelles. Scale bare $=500 \mathrm{~nm}$. (b) Size distribution of MRC-PPL micelles based on dynamic light scattering. (c) Zeta potentials of PSO, PPL, MRC-PPL micelles and MRC-PPL@PSO micelles. (d) UV-Vis absorbance. (e) Fluorescence intensity of MRC-PPL micelles and Cy5.5. (f) Fluorescence intensity of MRC-PPL micelles, without or with MMP-13 $(0.01 \mu \mathrm{M})$, in the absence or presence of MMP-13 inhibitor $(0.45 \mu \mathrm{M})$. (g) In vitro release of PSO from MRC-PPL micelles in PBS ( $\mathrm{pH} 6.5$ and 7.4) with $0.1 \%$ Tween 80 (mean \pm SD, $n=3$ ). 
$\mathbf{a}$

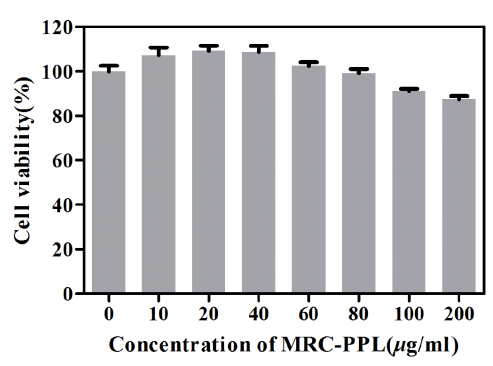

b

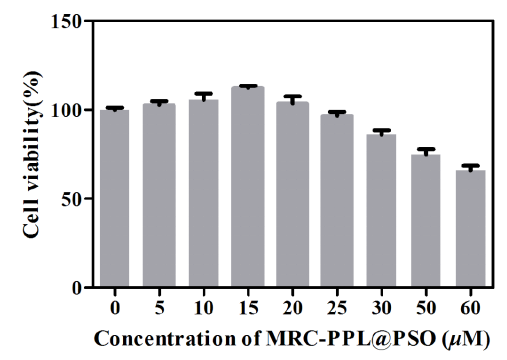

c

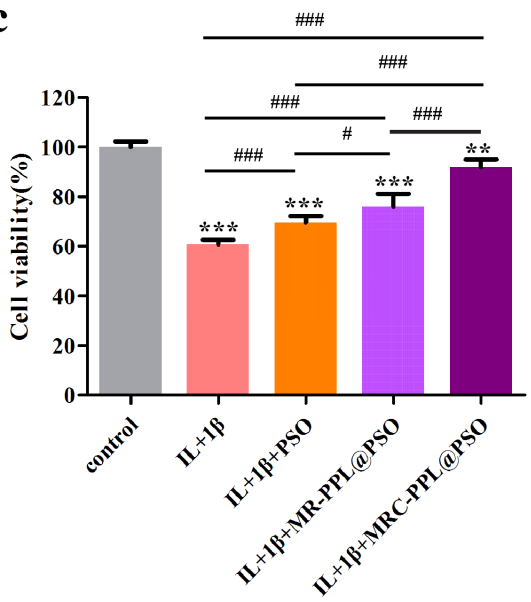

d

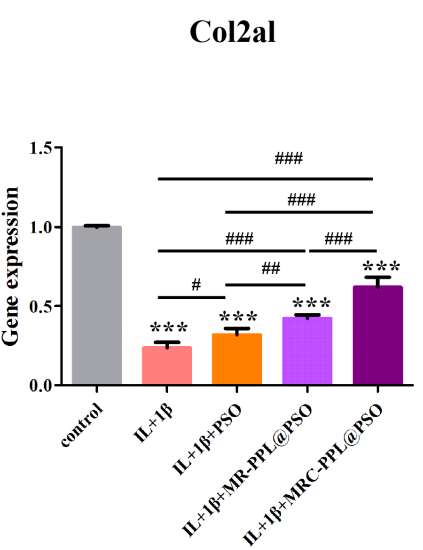

$\mathbf{e}$

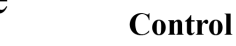

四
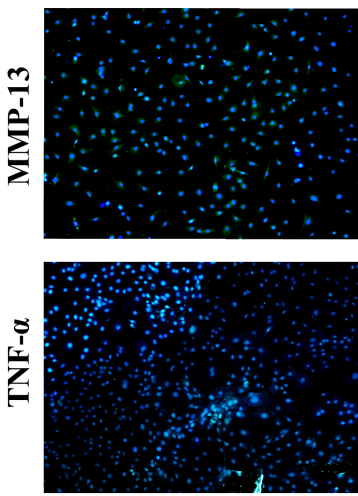
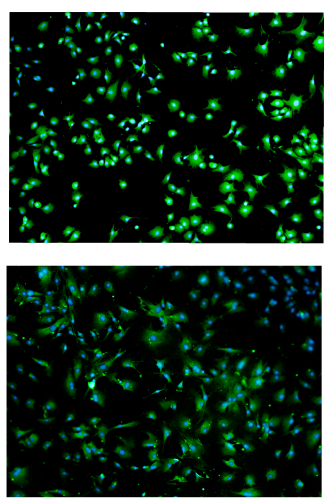
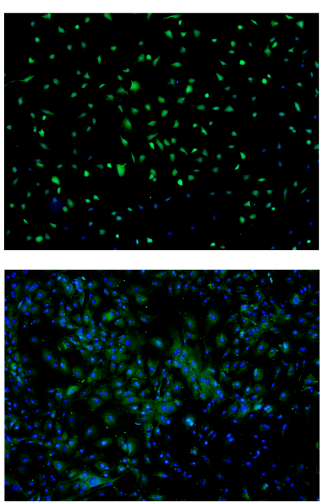

IL-1ß+PSO
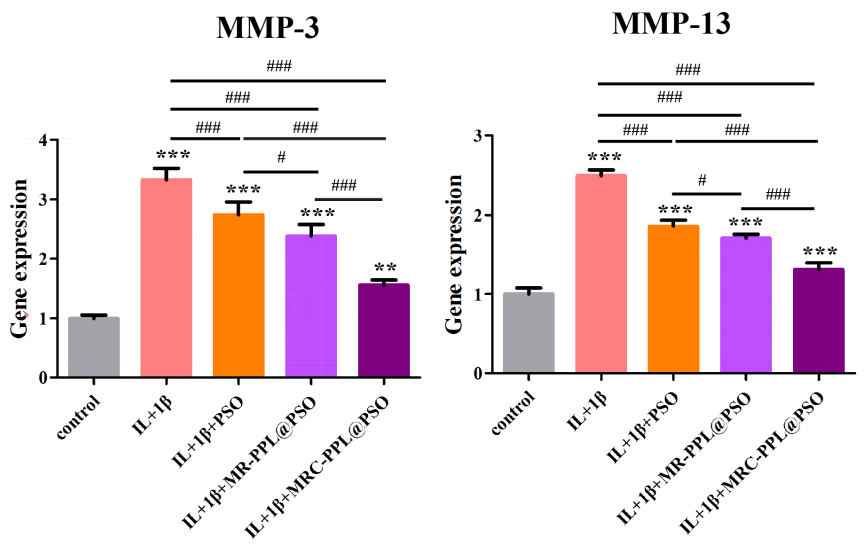

IL-1ß+MR-PPL@PSO

IL-1ß+MRC-PPL@PSO

\section{Figure 3}

In vitro study on chondrocytes isolated from C57BL6/J mice. ( $a$ and b) Cell viability after treatment with MRC-PPL or MRC-PPL@PSO. (c) Cell viability after various treatments to IL-1 $\beta$-stimulated chondrocytes. (d) Relative mRNA levels of Col2a1, TNF-a, MMP-3 and MMP-13 on IL-1 $\beta$-stimulated chondrocytes with various treatments. (e) HE staining and immunofluorescence images. The nuclei were counterstained with DAPI (blue), and MMP-13 or TNF-a positive staining was stained with FITC (green). Scales bar: 400 
$\mu \mathrm{m}$. Each data point represents mean $\pm \mathrm{s.d}(\mathrm{n}=3) .{ }^{*}$, \# indicate $\mathrm{p}<0.05 ; * \star$, \#\# indicate $p<0.01 ; \star \star \star$, \#\#\# indicate $p<0.001$.

$\mathbf{a}$

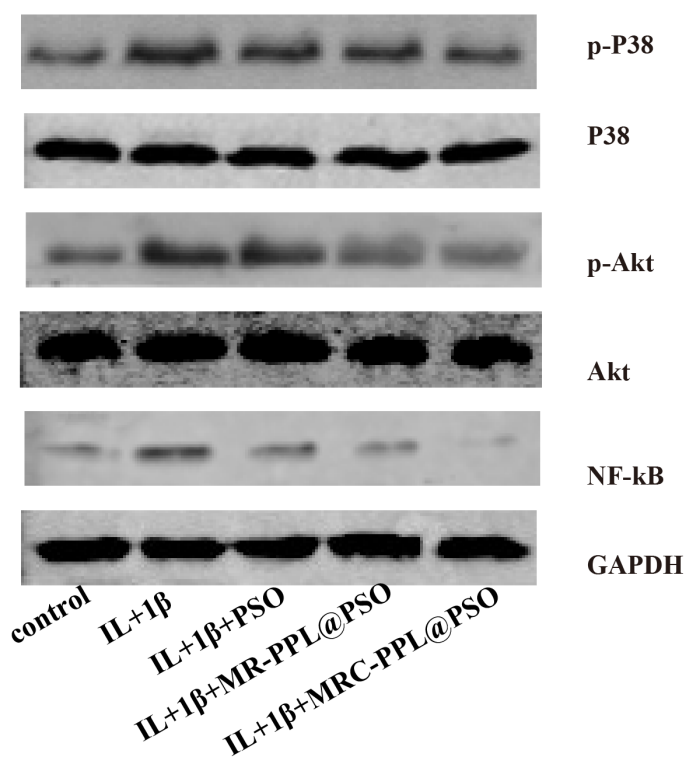

c

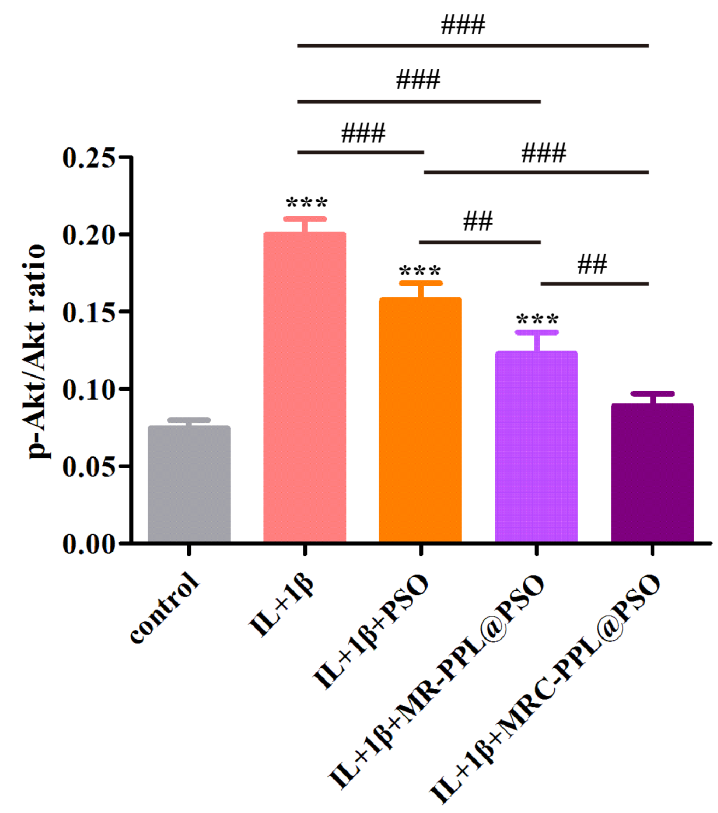

b

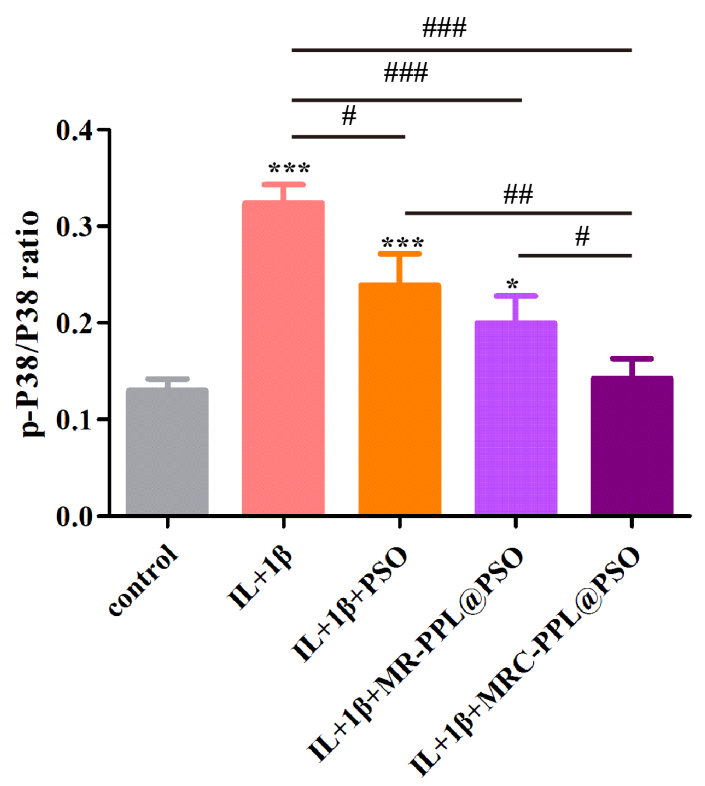

d

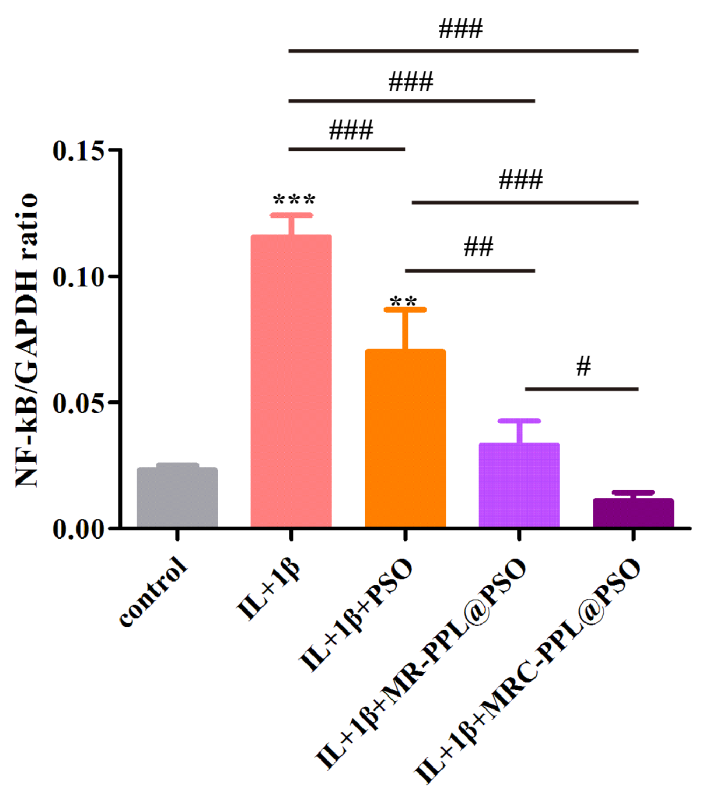

Figure 4

Molecular mechanism of MRC-PPL@PSO. The expression of PI3K/AKT, MAPK and NF-KB signaling pathway proteins p-P38, P38, p-Akt, Akt and NF-KB was determined by (a) western blot and (c-d) quantification analysis. Each data point represents mean \pm s.d $(n=3)$. ${ }^{*}$, indicate $p<0.05$; $* *$, \#\# indicate $p<0.01 ; * \star \star$, \#\#\# indicate $p<0.001$. 

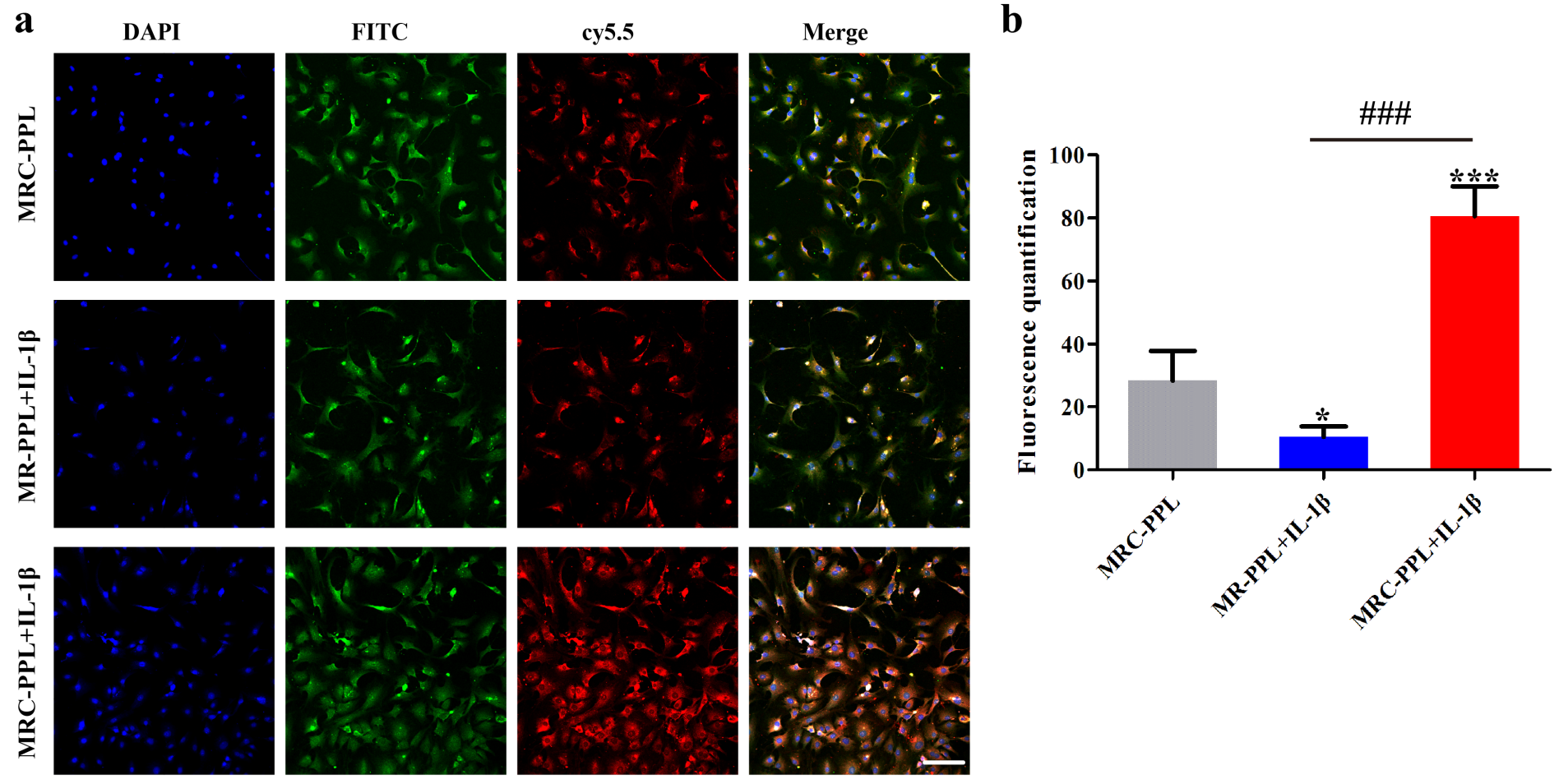

Figure 5

In vitro cellular uptake of MRC-PPL or MR-PPL micelles. (a) Immunofluorescence staining in chondrocytes to co-localized with collagen type II in the presence of MMP-13 or its inhibitor. The nuclei were counterstained with DAPI (blue) and collagen type II was stained with FITC (green). Scales bar: 400 $\mu \mathrm{m}$. (b) Fluorescence quantification of Cy5.5 after uptake of MRC-PPL or MR-PPL micelles by cells. Scale bars: $40 \mu \mathrm{m}$. $(\mathrm{n}=3$; mean \pm s.d; $*$, \# indicate $\mathrm{p}<0.05, * \star$, \#\# indicate $\mathrm{p}<0.01, * \star *$, \#\#\# indicate $\mathrm{p}<0.001$.) 

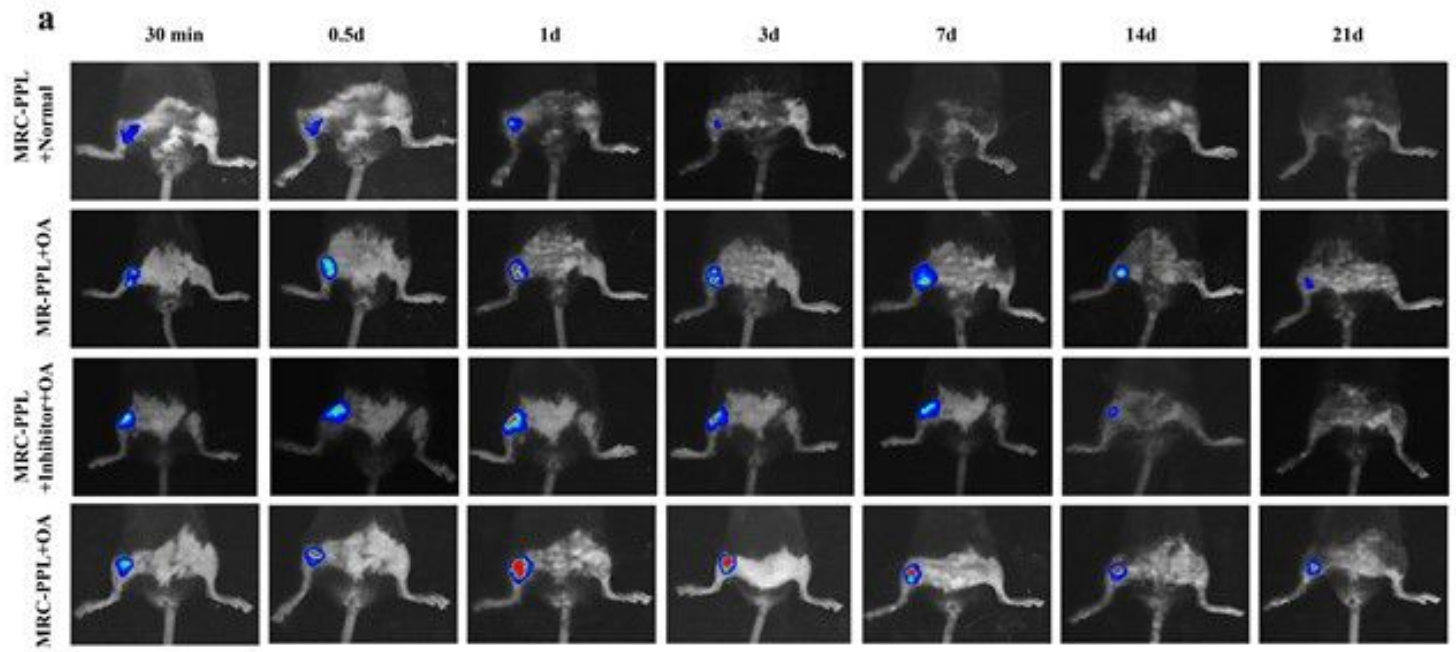

b

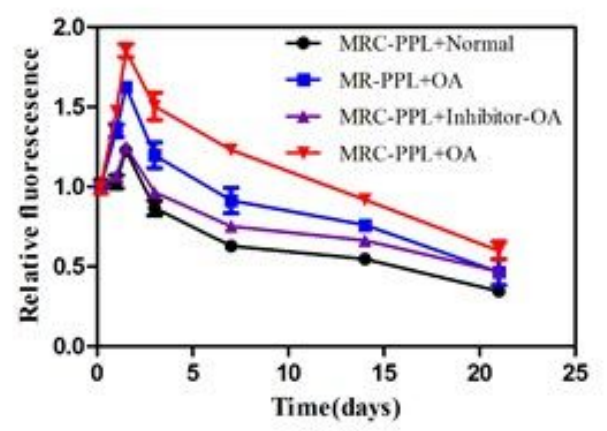

c
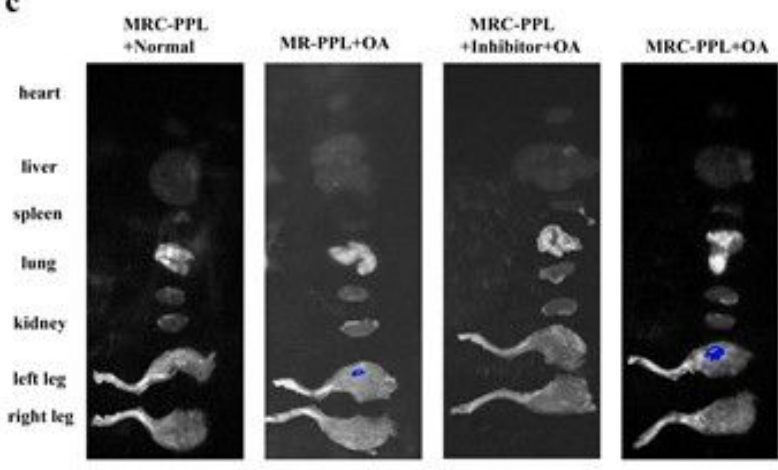

Figure 6

In vivo fluorescence imaging in normal or OA knees of mice. (a) Fluorescence imaging of OA joints injected with MRC-PPL, MRC-PPL+MMP-13 inhibitor, or MR-PPL at different time post-IA injection, as well as normal joint injected with MRC-PPL (excitation $=630 \mathrm{~nm}$, emission $=700 \mathrm{~nm}$ ). (b) The corresponding fluorescence intensity from OA joints at different times. (c) Ex vivo fluorescence imaging of heart, liver, spleen, lung, kidney, left knee and right knee at day 21 post-injection. $(n=5$, mean \pm s.d.). 
a

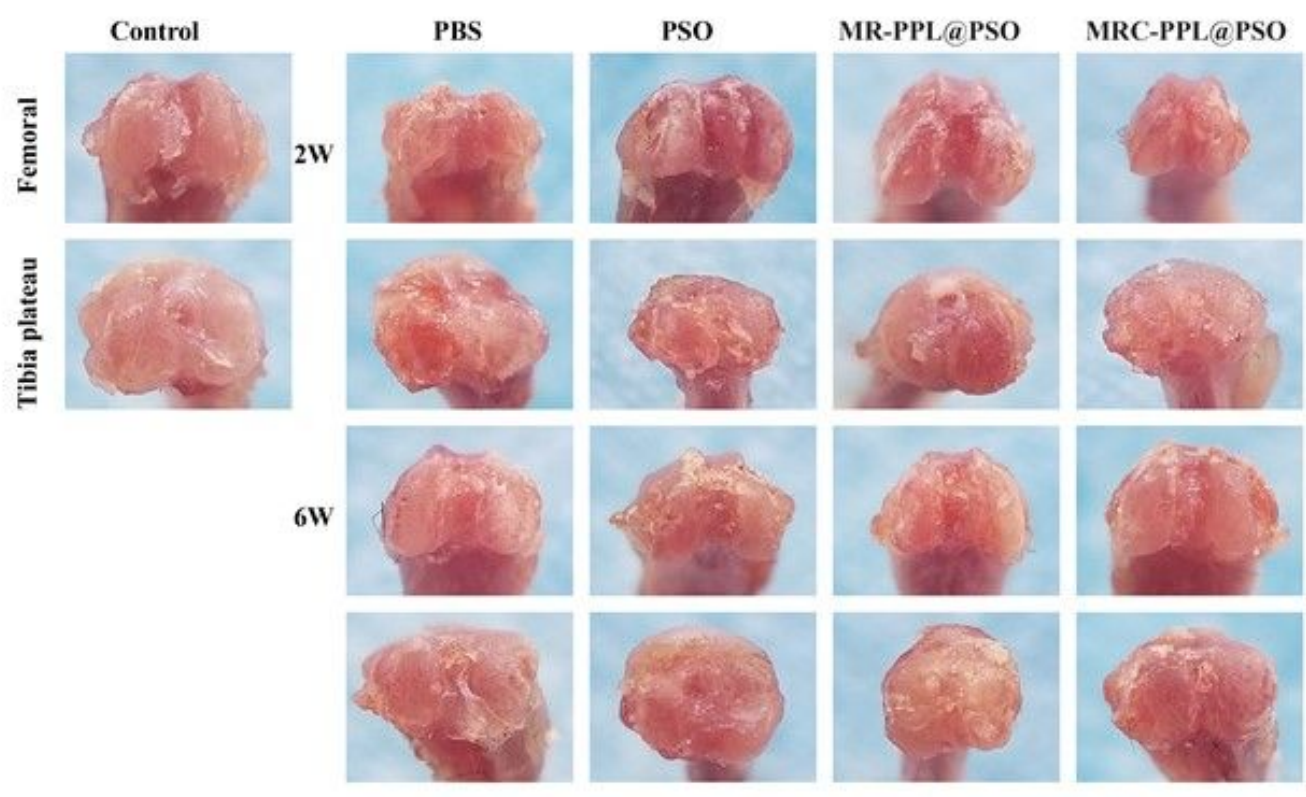

b
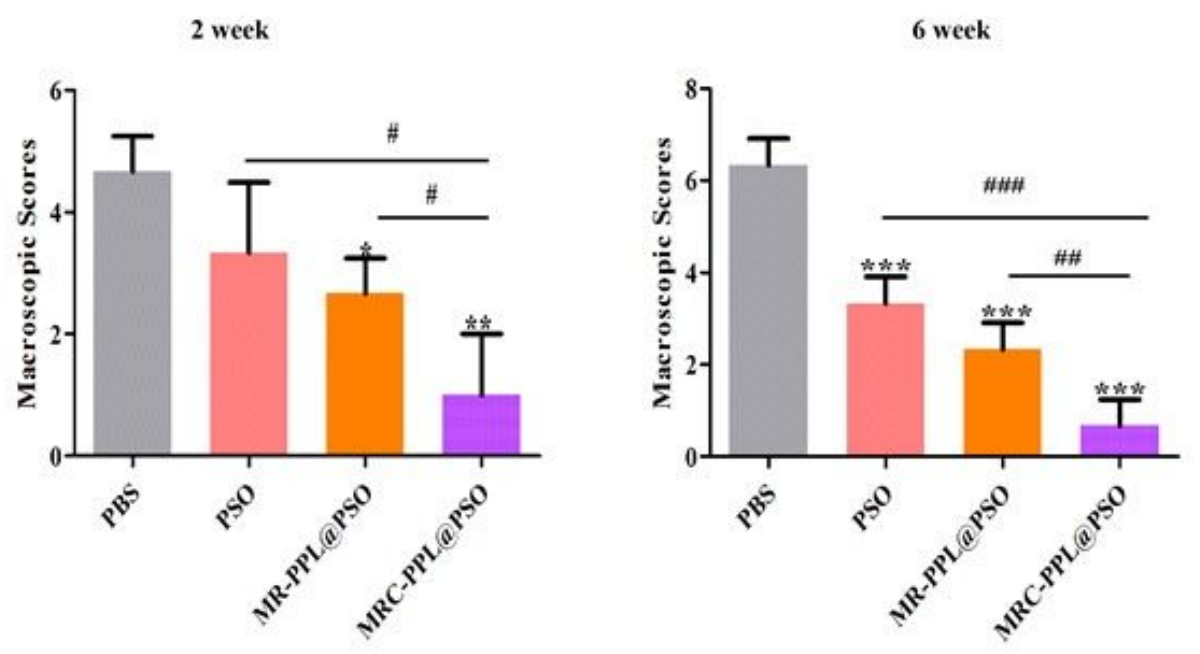

\section{Figure 7}

The macroscopic observation (a) and macroscopic score (b) of cartilage after IA-injection with PBS, PSO, MR-PPL@PSO and MRC-PPL@PSO for 2 and 6 weeks. Each data points represents mean \pm s.d. $(n=5) . *$, \# indicate $p<0.05 ; * \star$, \#\# indicate $p<0.01 ; * \star *$, \#\#\# indicate $p<0.001$. 
a
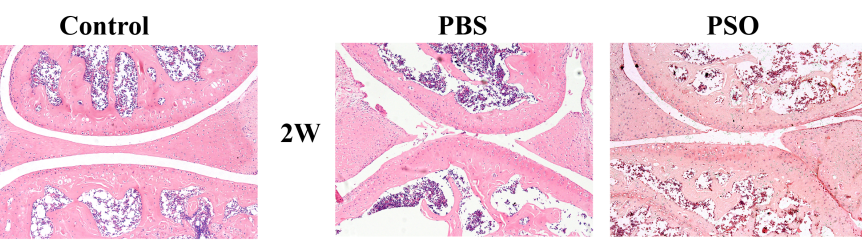

MR-PPL@PSO

MRC-PPL@PSO

6W
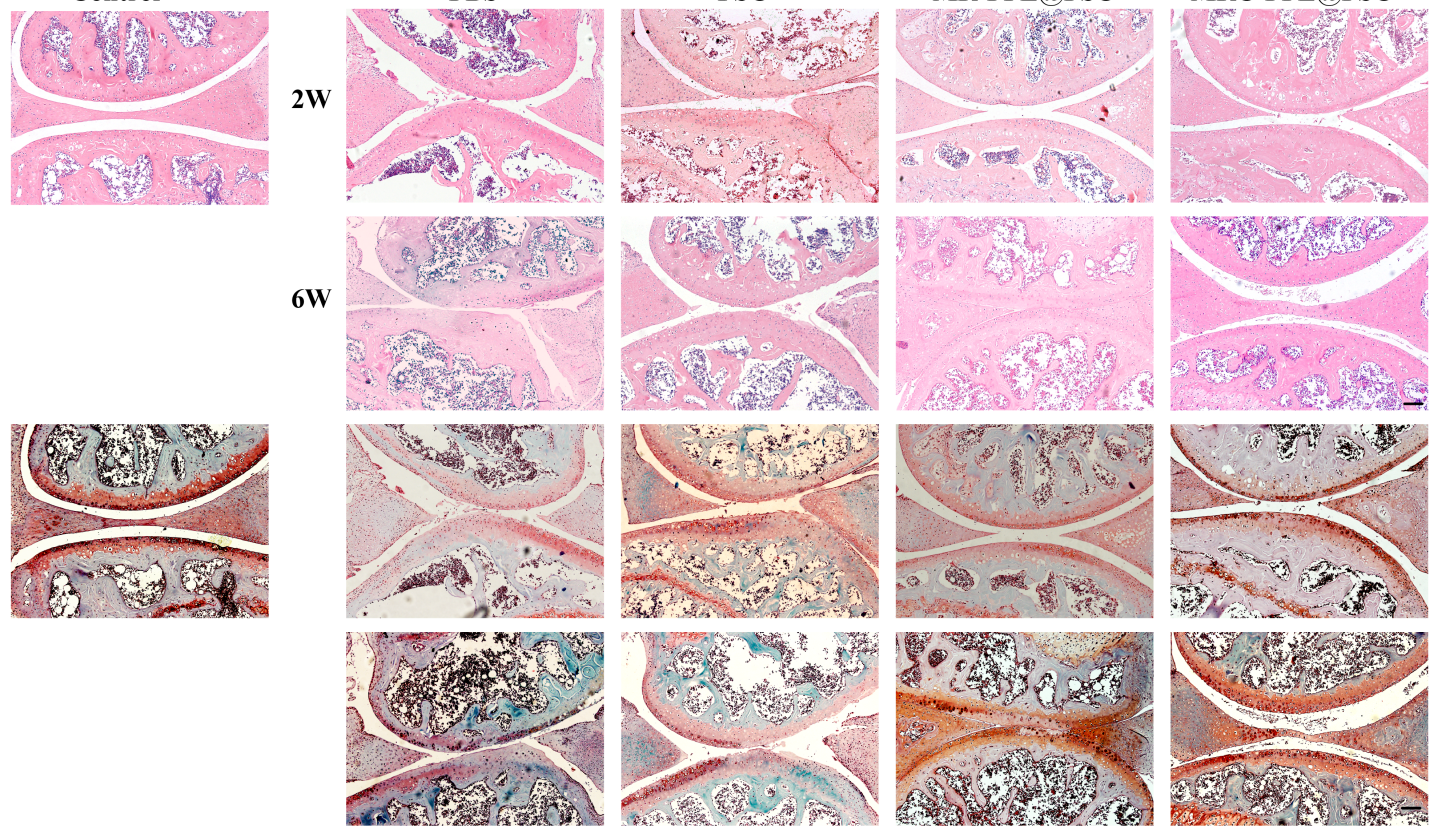

b

2 week
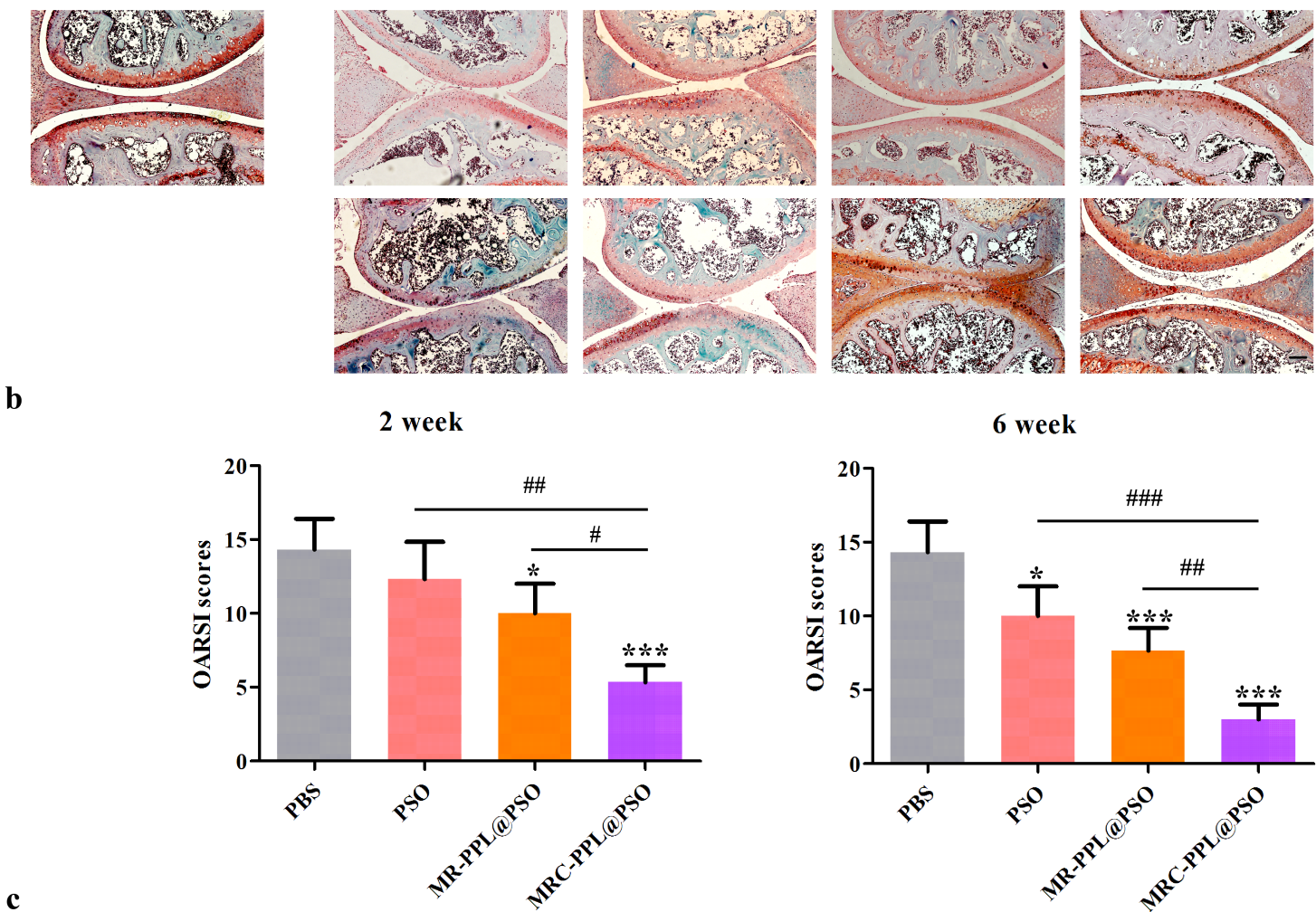

6 week
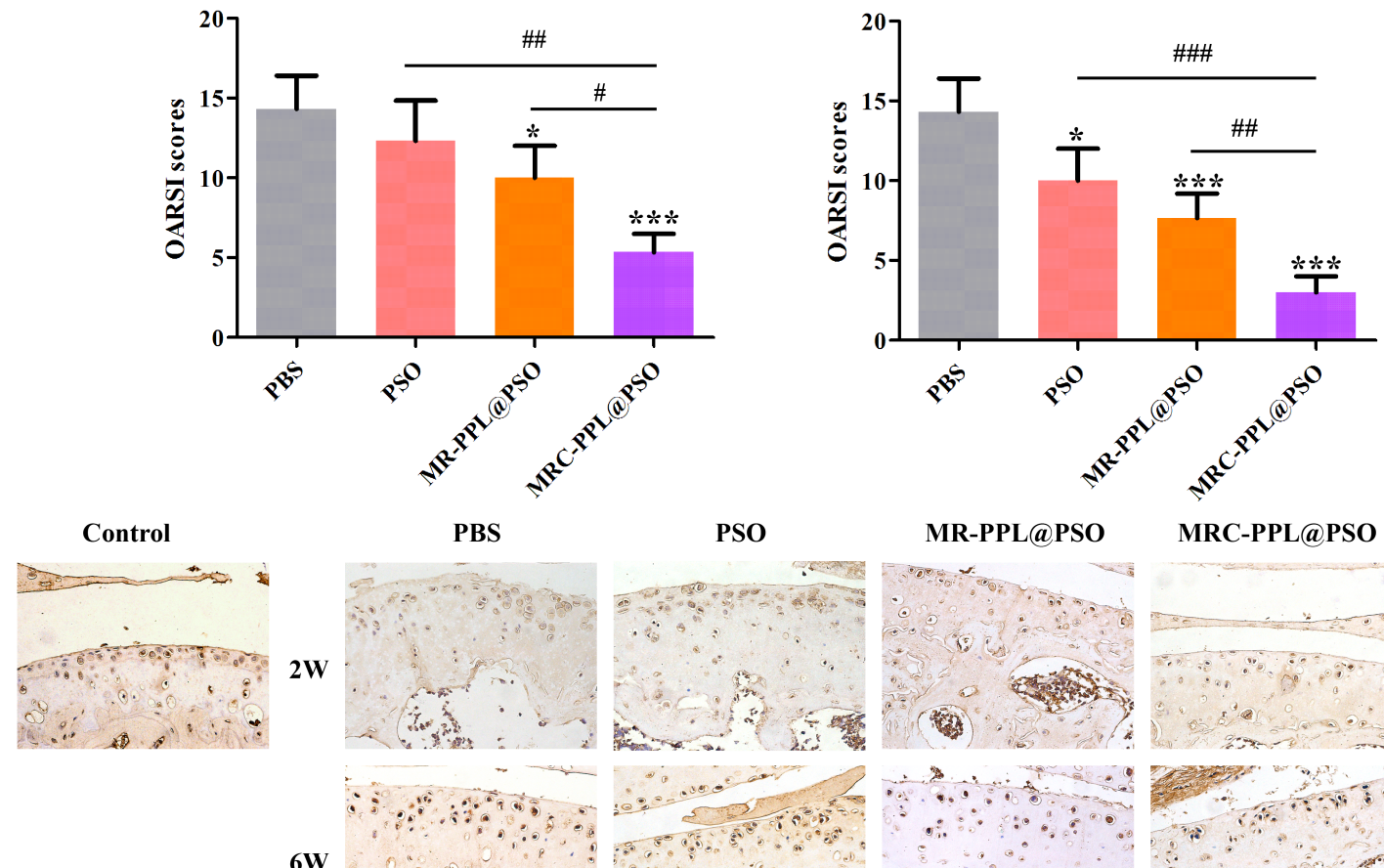

6W

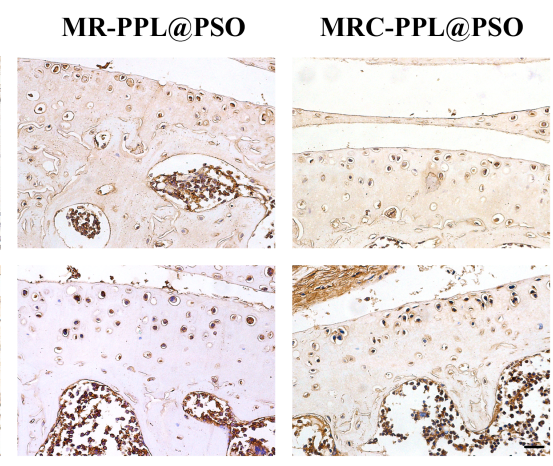

\section{Figure 8}

Histological analyses of different treatments for 2 or 6 weeks. (a) H\&E (upper) and safranin-O/fast green staining (lower) of cartilage sections after the treatments with PBS, PSO, MR-PPL@PSO and MRCPPL@PSO. Scale bar =1mm. (b) OARSI scores of articular cartilage after the treatments. Each data points represents mean \pm s.d. $(n=5) .{ }^{*}$, \# indicate $p<0.05 ; * \star$, \#\# indicate $p<0.01$; $* \star *$, \#\#\# indicate $p<$ 
0.001. (c) Immunohistochemical staining of MMP-13 on cartilage sections after the treatments. Scale bar $=300 \mu \mathrm{m}$. 